\title{
ESTUDIO COMPARATIVO DE BIOFERMENTOS NO ENRIQUECIDOS Y ENRIQUECIDOS, OBTENIDOS EN DIGESTORES TIPO BATCH Y SEMI-CONTINUOS
}

\section{COMPARATIVE STUDY OF NON-ENRICHED AND ENRICHED BIOFERMENTS, OBTAINED IN BATCH AND SEMI-CONTINUOUS DIGESTERS}

Recibido: 24 de septiembre 2021

Aceptado:6 de noviembre 2021
C. Mojica Mesinas ${ }^{1}$

D. C. Acosta Pintor ${ }^{2}$

E. Vidal Becerra ${ }^{3}$

A. L. Hernández Medina ${ }^{4}$

\section{RESUMEN}

El propósito del presente trabajo es realizar un estudio comparativo de los bioles enriquecidos y no enriquecidos para apoyar al sector agropecuario al estudiar el aprovechamiento del estiércol generado en sus ranchos y sus sub-productos (biogás, biol y biosol), así como proponer alternativas para un mejor manejo sanitario, dada la alta carga patógena que tiene el estiércol y que de usarse de manera directa pone en riesgo la inocuidad de las cosechas y la salud de los trabajadores. Para ello, se evaluó la calidad de cuatro (4) biofermentos producidos a través de procesos microbiológicos. El primero de ellos fue a nivel campo con un productor ganadero, en biodigestor tipo bolsa, sin enriquecer; El segundo fue a nivel laboratorio en biodigestión tipo batch con matraces kitazatos enriquecido; El tercer bioproceso se realizó a nivel laboratorio con cubetas de 20 litros, con la receta de Restrepo-Hensel para biofermentos enriquecidos; El cuarto biofermento se realizó a nivel laboratorio en un biodigestor semi-continúo del tipo Taiwán, con receta modificada de Restrepo-Hensel.

PALABRAS CLAVE: biofermento, Biol, enriquecido, digestor, batch, semicontinuo

\section{ABSTRACT}

The purpose of this work is to carry out a comparative study of enriched and non-enriched bioles to support the agricultural sector by studying the use of manure generated in their ranches and its byproducts (biogas, biol and biosol), as well as proposing alternatives for better sanitary management, given the high pathogenic load that manure has and that, if used directly, puts the safety of crops and the health of workers at risk (Peralta Veran, Juscamaita Morales, \& Meza Contreras, 2016). For this, the quality of four (4) bioferments produced through microbiological processes was evaluated. The first of them was at the field level with a livestock producer, in bag-type biodigester, without enrichment; The second was at the laboratory level in batch type biodigestion with enriched kitazate flasks; The third bioprocess was carried out at the laboratory level with 20 -liter buckets, with the Restrepo-Hensel recipe for enriched bioferments; The fourth bioferment was carried out at the laboratory level in a semi-continuous biodigester of the Taiwan type, with a modified Restrepo-Hensel recipe.

KEY WORDS: Bioferment, biol, enriched, digester, batch, semi-continuous

\footnotetext{
${ }^{1}$ Profesor del programa de Ingeniería Ambiental. Tecnológico nacional de México campus Ciudad Valles. cuitlahuac.mojica@tecvalles.mx

${ }^{2}$ Profesora del programa de Ingeniería Industrial. Tecnológico nacional de México campus Ciudad Valles. dulce.acosta@tecvalles.mx

${ }^{3}$ Profesora del programa de Ingeniería Industrial. Tecnológico nacional de México campus Ciudad Valles. elia.vidal@tecvalles.mx

${ }^{4}$ Alumna del programa de Ingeniería Ambiental. Tecnológico nacional de México campus Ciudad Valles. 16690356@tecvalles.mx
} 


\section{INTRODUCCIÓN}

Carhuancho León en el 2012, menciona que los biofertilizantes en forma líquida se pueden usar como fertilizante foliar, directo al suelo o como biocida para desinfestar las semillas y para acelerar el enraizamiento (Carhuancho, 2012).

Uno de los grandes retos de la agricultura, ante el importante incremento de la población mundial en los últimos años, es el abasto de alimentos en cantidad y calidad. Para enfrentar este reto, existen dos caminos posibles: Incrementar la superficie de cultivo e Incrementar los rendimientos por superficie (Aparcana et al, 2008).

El primero es una posibilidad cada vez más limitada, porque implica afectar otros ecosistemas. El segundo, requiere proporcionar a los suelos y/o a las plantas, fuentes de nutrientes adicionales en formas asimilables. Bajo el modelo de agricultura actual, esto último implica enormes aportes de insumos que se han vuelto "imprescindibles", como son los fertilizantes inorgánicos (Kinck, 1988).

El uso indiscriminado de insumos produce efectos muy negativos. El mayor problema es la contaminación del agua subterránea producida por el nitrógeno que se añade en forma de nitratos. Los nitratos aportados al suelo se mueven fácilmente a través de él, y debido a que son muy solubles en agua, pasan a las aguas subterráneas y permanecen en ellas durante años, y, además, el aporte de nitrógeno a lo largo del tiempo tiene un efecto acumulativo. Otro fertilizante de amplio uso, la urea, libera amoniaco en su proceso de descomposición. Una parte del amoniaco pasa a la atmosfera contribuyendo a la lluvia ácida y otra parte contamina el agua subterránea. Así mismo, a parte de los daños al agua y al ambiente, también hay daños al suelo y a la salud (CORPOICA, 2008).

Por lo anteriormente expuesto, surge la necesidad de investigar, analizar y de ser posible recomendar tratamientos sencillos y que impliquen pocos recursos para valorizar el estiércol bovino generado en los ranchos y dejar de verlo como un residuo problema al convertirlo en un fertilizante orgánico enriquecido, seguro para los cultivos de caña, que es el cultivo dominante en la región y de esta manera reducir las cargas al ambiente por el uso excesivo de fertilizantes químicos (Restrepo-Hensel, 2013).

\section{METODOLOGÍA}

\section{Producción de biol en campo}

Para tener un punto de referencia de los bioles, que se producen en los biodigestores, a nivel productor ganadero, se alimentó un biodigestor tipo Taiwán durante un periodo de 35 días, para ello, se usó como materia prima estiércol fresco de ganado bovino, con un $\mathrm{pH}$ inicial de 6.5 y un contenido de sólidos totales de 10.85. Se trabajó con un biodigestor semicontinuo,
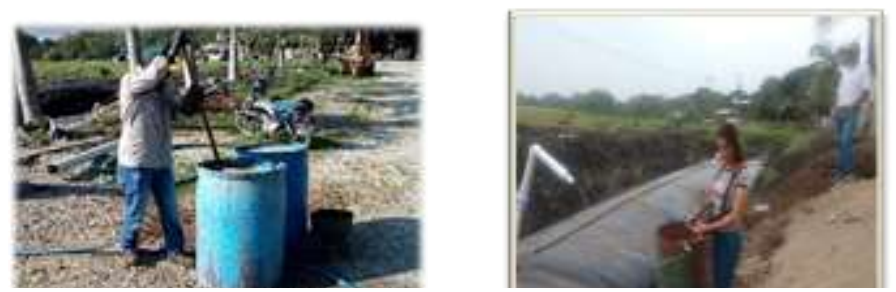

Figura 1. Preparación de la mezcla y alimentación al biodigestor para la producción del biol en campo. Fuente propia 
tipo Taiwán donde se llevó a cabo el proceso de digestión anaeróbica (Ver figura 1). Una vez que salió biol de proceso de fermentación del biodigestor, se tomó una muestra y se envió para su análisis al laboratorio Fertilab y con la información de los análisis fisicoquímicos de los mismo se consideran como datos de inicio (Restrepo-Hensel, 2013).

\section{Producción de biofermento en matraces Kitazato de 2 litros}

El biofermento se produjo en el mes de octubre de 2020, a partir de una mezcla de tres materias primas: Un biol (obtenido previamente de un biodigestor bajo condiciones de campo), leche de vaca y jugo de caña; esto a fin de aumentar la sinergia de la fermentación y obtener una buena disponibilidad de los nutrientes para las plantas y el suelo. Cabe mencionar que el biol previo se produjo en el Rancho ganadero "El Gallito", ubicado en Ciudad Valles, S.L.P (García, 2020).

Para la producción del biofermento, fueron necesarios tres matraces Kitasato de 2 litros de capacidad. La mezcla de las materias primas citadas, se definió tomando como referencia una de las recetas de Restrepo-Hensel en 2013 y los resultados de otros trabajos (MedinaQuipuzco-Juscamaita, 2021). De acuerdo con lo establecido por Restrepo-Hensel, 2013; los materiales e ingredientes para preparar los biofertilizantes que se presentan a continuación son los más sencillos, para ser aplicados en cualquier cultivo y pueden ser preparados por cualquier productor en cualquier lugar. La mezcla quedo de la siguiente manera: Biol obtenido en campo directamente de un biodigestor semicontinuo (70\%) con un volumen de 1.05 litros, más Jugo de caña $(20 \%)$ con volumen de 0.3 litros y leche de vaca (10\%) con un volumen de 0.15 litro (Restrepo-Hensel, 2013).

A los matraces Kitazato se les conectó por el tubo lateral una manguera que a la vez se introdujo en un frasco con agua, para ser utilizado como sistema de liberación de los gases (ver figura 2).

Una vez vertidas las mezclas a los matraces, comenzó la etapa de proceso de producción en

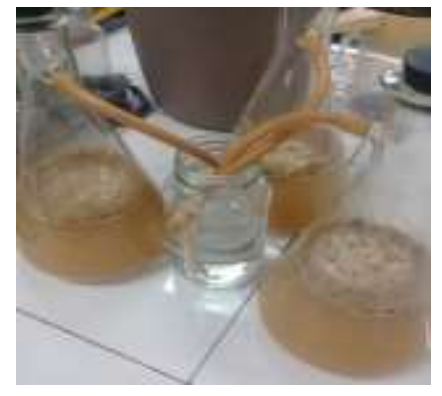

Figura 2. Preparación de matraces para producción del primer biofermento

un periodo inicial de cinco días, durante este tiempo se midieron y registraron datos de $\mathrm{pH}$, porcentaje de ácido láctico y conductividad eléctrica (Ver figura 3). 

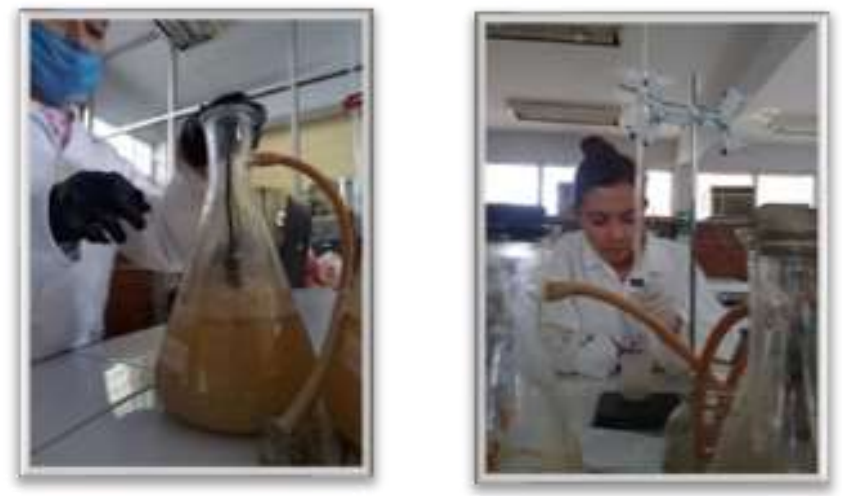

Figura 3. Mediciones al primer biofermento

Transcurridos los cinco días, comenzó una etapa de estabilidad del producto por 30 días. La medición del pH y de la conductividad eléctrica se realizó con un multiparamétrico, marca OAKTON, modelo PCD650. El porcentaje de ácido láctico se determinó a través del ácido láctico titulable, mediante la metodología de la titulación con hidróxido de sodio $0.1 \mathrm{~N}$ hasta conseguir el punto en que se presentó el cambio de $\mathrm{pH}$ del indicador fenolftaleína (medición indirecta) (Vidal et alt, 2020).

Producción del biofermento receta de Restrepo-Hensel (en recipientes plásticos de 20 litros)

La producción de este biofermento, se realizó en los meses de septiembre y octubre del 2020 y se usaron como biodigestores tipo Batch, tres recipientes plásticos con tapas, de 20 litros de capacidad. Como materia prima se usó estiércol fresco de bovino, ceniza de fogón, melaza, suero y agua (Hernández, 2021).

Se prepararon los biodigestores. Para ello se les hicieron orificios a las tapas de los recipientes donde se ubicó el cople y un tramo de manguera, mismos que fueron sellados con silicón para evitar fugas (Ver figura 4).

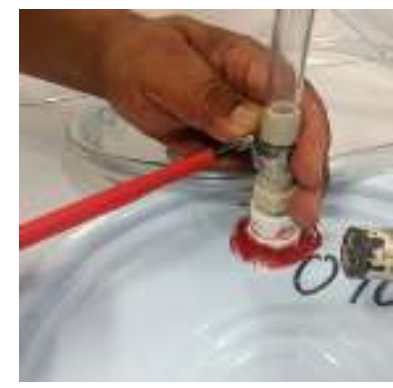

Figura 4. Preparación de los biodigestores tipo Batch para la producción del segundo biofermento 
Enseguida, se preparó la mezcla para el biofermento utilizando 14 lts de agua, $5 \mathrm{~kg}$ de estiércol bovino, $400 \mathrm{gr}$ de ceniza, $200 \mathrm{ml}$ de melaza y $400 \mathrm{ml}$ de suero (Ver figura 5).

Para empezar la elaboración de la mezcla, primero se pesó cada uno de los componentes; posteriormente se colocó el estiércol y la ceniza en el recipiente de plástico que haría la función de biodigestor y ahí se disolvió con 10 litros de agua mediante agitación constante. Por separado, en otro recipiente se disolvió en un litro de agua la melaza conjuntamente con el suero; una vez bien diluidos, se vertió esta mezcla para revolverla con el estiércol. Para finalizar, se agregó el agua al recipiente plástico sin llenarlo completamente, dejando un espacio de aproximadamente $3 \mathrm{~cm}$ entre el líquido y la tapa, ese espacio fue ocupado por gases que se forman durante el proceso de la fermentación. Es importante, no olvidar que en cada uno de los pasos del proceso de preparación del biofermento se debe agitar continuamente (Hernández Medina, 2021).

Como puede observarse, los biodigestores se cerraron herméticamente para el inicio de la
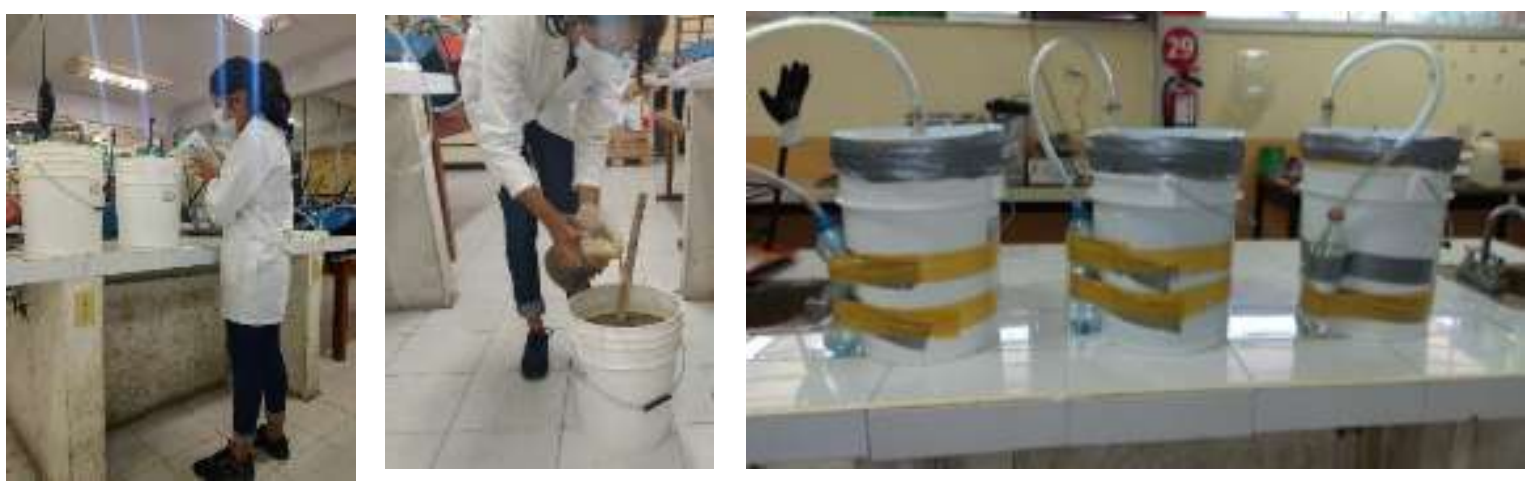

Figura 5. Preparación de la mezcla y montaje de los biodigestores para la producción del segundo biofermento

fermentación anaeróbica del biofertilizante, se les adhirió una botella de plástico con agua, conectada del biodigestor a través de una manguera, como sistema de salida de los gases (sello de agua) y fueron marcados para su fácil identificación. Se mantuvieron por un tiempo de 35 días en condiciones ambientales, con una media de temperatura de $28^{\circ} \mathrm{C}$ y presión atmosferica estándar (1 atm ó 760 $\mathrm{mmHg}$ ). La temperatura ideal según Restrepo y Hensel, es la del rumen de las vacas, es decir que oscile entre 38 y $40{ }^{\circ} \mathrm{C}$ (Restrepo-Hensel, 2013).

El producto estuvo listo para ser utilizado como abono líquido aproximadamente a los 35 días, aunque en lugares con climas muy fríos, este tiempo puede incrementarse hasta los 60 o 90 días, por este motivo, el mejor indicador para saber si el biofermento ya está listo para ser utilizado, es observar que ya no se formen burbujas por la trampa de agua; es decir que ya no haya salida de gas. Otro indicador, pero en este caso de su calidad, es el aroma a fermento y el color ámbar (café muy claro) del líquido. Por el contrario, un olor a podredumbre y colores azulados indicarían que el proceso no se llevó a cabo adecuadamente, por lo tanto, el producto debe desecharse. Para evitar que esto suceda no debe de entrar aire al biodigestor durante el proceso de fermentación. En el caso del presente experimento, el producto presentó un olor agradable y color café claro. (Pacheco et alt, 2017) 
Para tratamientos foliares Restrepo y Hensel, (2013) recomiendan una concentración del 5\% al 10\%; es decir usar de 5 a 10 litros del biopreparado por cada 100 litros de agua que se vierten sobre los cultivos. Si se trata de aplicación manual, entonces por cada mochila aspersora de 20 litros, usar de 1 a 1 1/2 litros del biopreparado. Cuando se aplique directamente al suelo, ya sea por medio de una regadera o a boquilla abierta de la bomba de espalda, aumentar la concentración a 3 litros de producto por cada 20 litros de agua. Es necesario colar el biofermento para evitar que algún residuo de basura llegue a taponear la boquilla de la bomba de aspersión. Después de su elaboración, el biopreparado debe ser almacenado en un lugar fresco y ser utilizado lo más pronto posible, esto debido a que, con el paso del tiempo tiende a acidificarse; un producto viejo puede llegar a provocar la quema del follaje de las plantas (Restrepo-Hensel, 2013).

\section{Producción de biofermento en un biodigestor semicontinuo tipo Taiwán}

En tanto ocurre el proceso de fermentación en los digestores tipo Bach, se inicia la alimentación diaria del digestor semicontinuo tipo Taiwán, como materia prima se usó estiércol fresco de bovino, ceniza de fogón, melaza, suero y agua. Se realizó el cálculo para alimentar el biodigestor tipo Taiwán diariamente con una mezcla aproximadamente a un litro de los componentes anteriormente mencionados, obteniendo los siguientes datos: 0.250 kilogramos de excreta bovina, 0.020 kilogramos de ceniza, 0.010 litros de melaza, 0.020 litros de suero y 0.750 litros de agua (Hernández, 2021).

Previo a introducir la carga diaria al biodigestor se realizó toma de datos con un multiparamétrico, marca OAKTON, modelo PCD650, de las siguientes características químicas: $\mathrm{pH}$, conductividad, $\mathrm{NaCl}$, y DO (Ver figura 6).

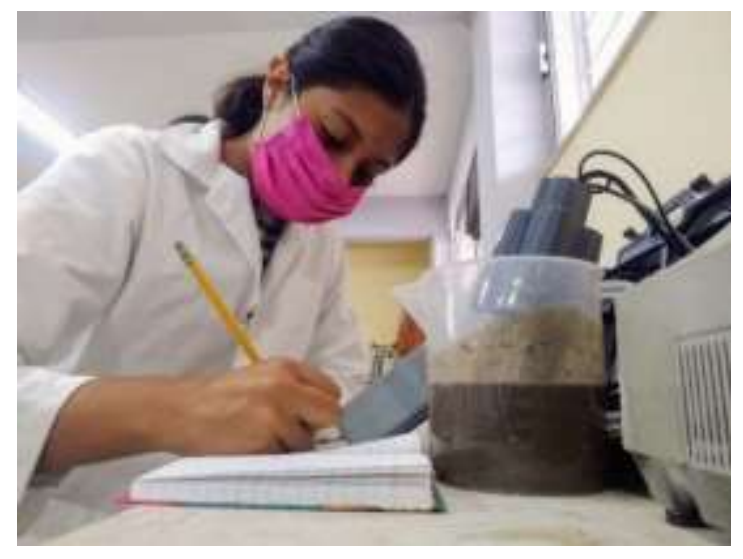

Figura 6. Medición de parámetros químicos

Se alimentó el biodigestor semi continuo por 30 días con la preparación de la mezcla, realizando las mediciones correspondientes y la anotación diaria de los parámetros.

Cumplido el tiempo de alimentación y retención, una vez que hay salida de biol, se realizó la toma de la muestra para su análisis en el laboratorio Fertilab.

\section{Recolección de las muestras de biofermento para los análisis de laboratorio}

Respecto al biol producido en campo, se tomó la muestra directamente de la salida del biodigestor, enviando la muestra para su análisis en el laboratorio de Fertilab. 
En el caso del primer biofermento (en matraces Kitazato de 2 litros), al terminar la etapa de estabilidad (después de los 35 días de fermentación en los matraces), se procedió a la toma de muestra; para ello se mezclaron los tres matraces y de esta mezcla se tomó un litro de

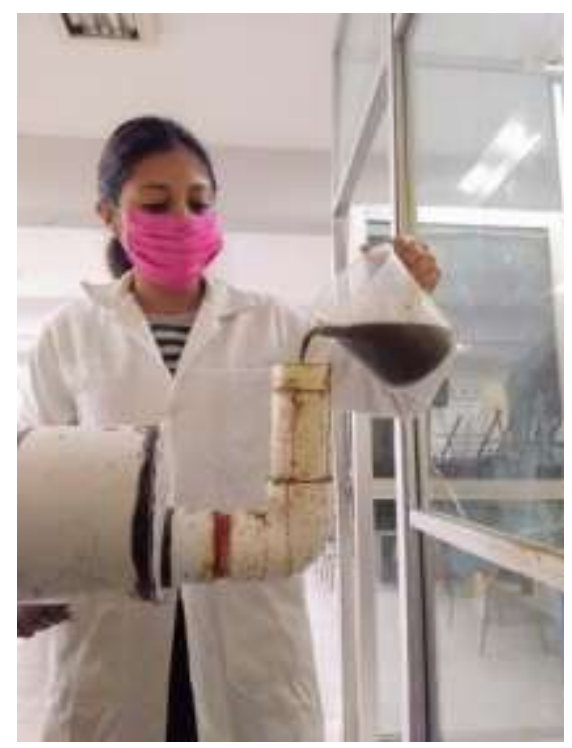

\section{Figura 7. Alimentación de biodigestor semicontinuo}

muestra, que se envió al laboratorio externo (Fertilab), para las pruebas correspondientes de micro y macronutrientes (Restrepo-Hensel, 2013).

En el caso del segundo biofermento (en recipientes de plástico de 20 litros), transcurridos los 35 días, cuando ya no hubo formación de burbujas en la botella de salida de los gases, se procedió a tomar la muestra. Para ello, se tomó de cada recipiente ("biodigestor") una muestra de un litro, que se enviaron por separado al laboratorio Fertilab (Hernández, 2021).

Para el tercer biofermento (biodigestor semicontinuo), al transcurrir los 30 días de alimentación y al salir biol como excedente (tiempo de retención hidráulica), se tomó un litro de muestra, el cual fue enviado al laboratorio Fertilab (Op. Cit, pág. 37).

\section{Análisis físico-químicos}

Para los cuatro (4) biofermentos, los análisis de laboratorio, correspondientes a las pruebas físico químicas de humedad, materia orgánica, cenizas, conductividad eléctrica $(\mathrm{CE}), \mathrm{pH}$, Cationes básicos como Calcio (Ca), Magnesio (Mg), Sodio (Na), Potasio (K), y Azufre (S); micronutrientes Hierro (Fe), Manganeso (Mn), Zinc ( $\mathrm{Zn})$, Cobre $(\mathrm{Cu})$, Boro (B) y Fosforo $(\mathrm{P})$, se realizaron en el laboratorio Fertilab, que es un laboratorio externo. La determinación de sólidos totales se realizó en el Laboratorio de Química del TecNM, campus Ciudad Valles.

Los métodos usados en el laboratorio fueron los que se presentan en la tabla 1 y los resultados se presentan en las tablas 2 y 3 , ubicada en la sección de resultados.

Tabla 1. Métodos usados en las pruebas físico químicas realizadas a biofermentos

\begin{tabular}{|l|l|}
\hline Determinación & Método \\
\hline $\mathrm{pH}$ & NMX-FF-109-SCFI-2007 \\
\hline
\end{tabular}




\begin{tabular}{|l|l|}
\hline Determinación & Método \\
\hline Cond. Eléctrica & NMX-FF-109-SCFI-2007 \\
\hline$*$ Nitrógeno total & Dumas \\
\hline$*$ Fósforo (P) & Digestión en microondas/ ICP \\
\hline$*$ Potasio (K) & Digestión en microondas/ ICP \\
\hline$*$ Calcio $(\mathrm{Ca})$ & Digestión en microondas/ ICP \\
\hline$*$ Magnesio (Mg) & Digestión en microondas/ ICP \\
\hline$*$ Sodio (Na) & Digestión en microondas/ ICP \\
\hline$*$ Azufre (S) & $\begin{array}{l}\text { Digestión en microondas / } \\
\text { Turbidiometría }\end{array}$ \\
\hline$*$ Hierro (Fe) & Digestión en microondas/ ICP \\
\hline$*$ Cobre $(\mathrm{Cu})$ & Digestión en microondas/ ICP \\
\hline$*$ Manganeso (Mn) & Digestión en microondas/ ICP \\
\hline$*$ Zinc (Zn) & Digestión en microondas/ ICP \\
\hline$*$ Boro (B) & Digestión en microondas/ ICP \\
\hline Humedad & Método gravimétrico \\
\hline Solidos totales (St) & $\begin{array}{l}\text { Gravimétrico/ NMX-AA-034-SCFI- } \\
2015\end{array}$ \\
\hline$*$ Materia orgánica & $\begin{array}{l}\text { Calcinación/ NMX-AA-034-SCFI- } \\
2015\end{array}$ \\
\hline * Carbonizas & Balcinación/ NMX-AA-034-SCFI- \\
\hline
\end{tabular}

\section{RESULTADOS}

En la tabla siguiente, se muestras los diferentes datos obtenidos de los biofermentos de acuerdo con la forma en que se generan: 
Tabla 2. Resultados de laboratorio y valores de requerimientos de los macro y micro nutrientes para caña de azúcar

\begin{tabular}{|c|c|c|c|c|c|c|}
\hline Parámetro & $\begin{array}{l}\text { Biol "El } \\
\begin{array}{l}\text { Gallito" en } \\
\text { condiciones } \\
\text { de campo }\end{array} \\
{[\%]}\end{array}$ & 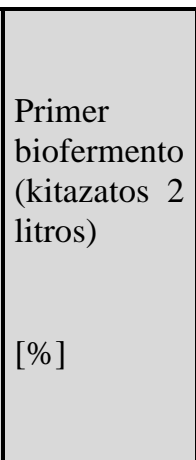 & $\begin{array}{l}\text { Biol II } \\
\text { (Cubetas } \\
20 \text { litros) } \\
\text { Restrepo- } \\
\text { Hensel } \\
{[\%]}\end{array}$ & $\begin{array}{l}\text { Resultado } \\
\text { biodigestor } \\
\text { Semi- } \\
\text { continúo } \\
\text { Enriquecido } \\
\text { [\%] }\end{array}$ & 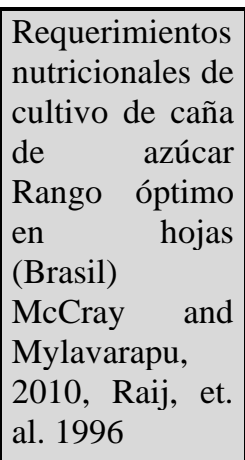 & Observación \\
\hline $\begin{array}{l}\text { Nitrógeno total } \\
\text { (N) }\end{array}$ & 0.1100 & 0.0022 & 0.0717 & 0.0300 & 1.80 a $2.50 \%$ & \begin{tabular}{|lr} 
Todos & los \\
biofermentos, se \\
encuentran muy \\
por debajo del \\
requerimiento
\end{tabular} \\
\hline Fósforo (P) & 0.0033 & 0.0001 & 0.0683 & 0.0037 & 0.15 a $0.30 \%$ & \begin{tabular}{|lr} 
Todos & los \\
biofermentos, & se \\
encuentran & por \\
debajo & del \\
requerimiento &
\end{tabular} \\
\hline Potasio (K) & 0.0800 & 0.0009 & 2.1712 & 0.1400 & 1.00 a $1.60 \%$ & $\begin{array}{|lr|}\text { Solo } & \text { el } \\
\text { biofermento } \\
\text { preparado de } \\
\text { acuerdo a } \\
\text { Restrepo-Hensel, } \\
\text { cumple con los } \\
\text { requerimientos, } \\
\text { los demás está } \\
\text { muy por debajo } \\
\text { del requerimiento. }\end{array}$ \\
\hline Calcio (Ca) & 0.0041 & 0.0002 & 1.4517 & 0.1800 & 0.20 a $0.80 \%$ & \begin{tabular}{|lr} 
Solo & el \\
biofermento \\
preparado de \\
acuerdo ror a \\
Restrepo-Hensel, \\
cumple con los \\
requerimientos y \\
se encuentra por \\
arriba; los demás \\
está muy por \\
debajo & del \\
requerimiento.
\end{tabular} \\
\hline Magnesio (Mg) & 0.0200 & 0.0004 & 0.3817 & 0.2700 & 0.10 a $0.30 \%$ & $\begin{array}{l}\text { El biofermento } \\
\text { preparado de } \\
\text { acuerdo a } \\
\text { Restrepo-Hensel } \\
\text { y el generado en } \\
\text { el biodigestor } \\
\end{array}$ \\
\hline
\end{tabular}




\begin{tabular}{|c|c|c|c|c|c|c|}
\hline Parámetro & $\begin{array}{l}\text { Biol "El } \\
\text { Gallito" en } \\
\text { condiciones } \\
\text { de campo } \\
\\
{[\%]}\end{array}$ & $\begin{array}{l}\text { Primer } \\
\text { biofermento } \\
\text { (kitazatos } 2 \\
\text { litros) } \\
\\
{[\%]}\end{array}$ & $\begin{array}{l}\text { Biol II } \\
\text { (Cubetas } \\
20 \text { litros) } \\
\text { Restrepo- } \\
\text { Hensel } \\
{[\%]}\end{array}$ & \begin{tabular}{|l} 
Resultado \\
biodigestor \\
Semi- \\
continúo \\
Enriquecido \\
{$[\%]$}
\end{tabular} & $\begin{array}{l}\text { Requerimientos } \\
\text { nutricionales de } \\
\text { cultivo de caña } \\
\text { de azúcar } \\
\text { Rango óptimo } \\
\text { en hojas } \\
\text { (Brasil) } \\
\text { McCray and } \\
\text { Mylavarapu, } \\
\text { 2010, Raij, et. } \\
\text { al. 1996 }\end{array}$ & Observación \\
\hline & & & & & & $\begin{array}{l}\text { semi continuo } \\
\text { cumplen con los } \\
\text { requerimiento. }\end{array}$ \\
\hline Sodio $(\mathrm{Na})$ & 0.0100 & 0.0002 & 0.0667 & 0.0400 & sin referencia & $\begin{array}{ll}\text { No existe } & \text { un } \\
\text { punto } & \text { de } \\
\text { referencia para } \\
\text { comparar los } \\
\text { resultados con las } \\
\text { necesidades } & \text { del } \\
\text { cultivo } & \end{array}$ \\
\hline Azufre (S) & 0.0082 & 0.0082 & 0.1217 & 0.2700 & 0.15 a $0.30 \%$ & $\begin{array}{l}\text { Solo } \\
\text { biofermento } \\
\text { generado en el } \\
\text { biodigestor semi } \\
\text { continuo cumplen } \\
\text { con los } \\
\text { requerimiento. }\end{array}$ \\
\hline Hierro(Fe) & 0.0010 & 0.3200 & 152.9000 & 0.2500 & 40 a $250 \mathrm{ppm}$ & \begin{tabular}{|l} 
Solo \\
biofermento \\
preparado de \\
acuerdo a \\
Restrepo-Hensel, \\
cumple con los \\
requerimientos y \\
se encuentra por \\
arriba; los demás \\
está muy por \\
debajo del \\
requerimiento.
\end{tabular} \\
\hline Cobre $(\mathrm{Cu})$ & 1.4900 & 1.2900 & 0.5283 & 20.1000 & 6 a $15 \mathrm{ppm}$ & $\begin{array}{l}\text { Solo } \\
\text { biofermento } \\
\text { generado en el } \\
\text { biodigestor semi } \\
\text { continuo cumplen } \\
\text { con los } \\
\text { requerimiento. }\end{array}$ \\
\hline
\end{tabular}




\begin{tabular}{|c|c|c|c|c|c|c|}
\hline Parámetro & $\begin{array}{l}\text { Biol "El } \\
\text { Gallito" en } \\
\text { condiciones } \\
\text { de campo }\end{array}$ & $\begin{array}{l}\begin{array}{l}\text { Primer } \\
\text { biofermento } \\
\text { (kitazatos } 2 \\
\text { litros) }\end{array} \\
{[\%]}\end{array}$ & $\begin{array}{l}\text { Biol II } \\
\text { (Cubetas } \\
20 \text { litros) } \\
\text { Restrepo- } \\
\text { Hensel } \\
{[\%]}\end{array}$ & \begin{tabular}{|l} 
Resultado \\
biodigestor \\
Semi- \\
continúo \\
Enriquecido \\
[\%]
\end{tabular} & 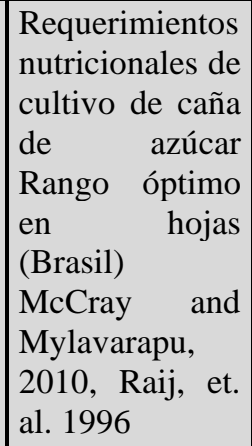 & Observación \\
\hline Manganeso(Mn) & 0.0500 & ND & 51.8333 & 0.2500 & 25 a $250 \mathrm{ppm}$ & \begin{tabular}{|l} 
Solo el \\
biofermento \\
preparado de \\
acuerdo a \\
Restrepo-Hensel, \\
cumple con los \\
requerimientos y \\
se encuentra en la \\
media; los demás \\
está muy por \\
debajo del \\
requerimiento.
\end{tabular} \\
\hline $\operatorname{Zinc}(\mathrm{Zn})$ & 0.1400 & 0.0600 & 20.1000 & 1165.0000 & 10 a $50 \mathrm{ppm}$ & $\begin{array}{l}\text { El biofermento } \\
\text { preparado de } \\
\text { acuerdo a } \\
\text { Restrepo-Hensel } \\
\text { y el } \\
\text { el } \text { generado en } \\
\text { semi codigestor } \\
\text { cumplen con los } \\
\text { requerimientos. } \\
\text { Pero el de } \\
\text { biodigestor } \\
\text { semicontinuo } \\
\text { puede ser toxico } \\
\text { por su alto } \\
\text { contenido de Zinc }\end{array}$ \\
\hline Boro(B) & 0.1800 & 1.3000 & 1.4400 & 305.0000 & 10 a $30 \mathrm{ppm}$ & \begin{tabular}{|l} 
Solo \\
biofermento \\
generado en el \\
biodigestor semi \\
continuo cumplen \\
con los \\
requerimiento y \\
se encuentra muy \\
por arriba, puede \\
presentar \\
problemas de \\
toxicidad por su \\
alto contenido de \\
Boro
\end{tabular} \\
\hline
\end{tabular}


De acuerdo con los resultados de los diferentes biofermentos, el Nitrógeno total (N) y Fosforo (P) se encuentran por debajo de los requerimientos nutricionales del rango óptimo en hojas, ninguno cumple con las necesidades para el cultivo de la caña de azúcar. (McCray \& Mylavarapu, 2021).

En cuanto a los requerimientos de Potasio (K), Calcio (Ca), Magnesio (Mg), Hierro (Fe), Manganeso (Mn) y Zinc (Zn); los biofermentos preparado de acuerdo a Restrepo-Hensel, cumple con los requerimientos nutricionales. En este biofermento se observa 6 de los elementos cumplen los requerimientos para un cultivo de caña de azúcar, es decir el 50\% de los elementos están dentro del rango óptimo de necesidades del cultivo. Sin embargo, para el Potasio (K) el indicador está ligeramente por arriba, sin que represente riesgo de toxicidad para el cultivo.

Para el biodigestor semi-continuo con alimentación enriquecida, los nutrientes que cumplen con las necesidades nutricionales, son: Magnesio (Mg), Azufre (S), Cobre (Cu), Zinc (Zn) y Boro (B). Para este biofermento se observa que 5 de los elementos están dentro del rango de necesidades de cultivo de caña de azúcar. Esto representa que un $41.6 \%$ de los elementos cumple con las necesidades totales del cultivo, el resto de los elementos están por debajo. Observando que el Zinc (Zn) y el Boro (B) presentan alta concentraciones, por lo que puede presentarse toxicidad en los cultivos.

Sólo dos (2) elementos de los nutrientes cumplen las necesidades nutricionales, tanto en los biofermentos preparados de acuerdo a Restrepo-Hansen y el biodigestor semi-continúo alimentado con una mezcla enriquecida; los cuales son: Magnesio (Mg) y Zinc (Zn). Los demás biofermentos están muy por debajo de las necesidades nutricionales que requiere un cultivo de caña. Recalcando que el biol enriquecido del biodigestor semi-continuo puede presentar toxicidad en el cultivo por el alto contenido de Zinc (Zn) y Boro (B).

Para el Sodio $(\mathrm{Na})$, no se encuentran referencias de las necesidades del es nutriente para el cultivo de caña de azúcar, por lo que no hay punto de referencia para su comparación.

Como se puede observar, la composición del biol enriquecido depende mucho del tipo de bioproceso en el cual se obtiene (Bacht o semi-continuo) y del tipo de receta que se utilice para alimentarlo;

\section{CONCLUSIONES}

De acuerdo con los resultados se observa que los biofermentos enriquecidos obtenidos en un digestor tipo Batch con un proceso homoláctico (Cubetas de 20 litros), Presenta el mejor resultado, al cumplir con el rango de necesidades nutricionales para un cultivo de caña de azúcar en 6 de 12 elementos medidos; lo que representa el 50\% de elementos.

Para el biodigestor semi-continuo tipo Taiwán con un proceso homoláctico y receta adaptada y enriquecida, se logra obtener el cumplimiento en 5 de 12 elementos medios, lo que representa el $41.6 \%$ de elementos que cumplen con el rango de las necesidades nutricionales para el cultivo de caña de azúcar. Presentando altos contenidos de Zinc (Zn) y Boro (B), que pueden presentar toxicidad en los cultivos.

Con respecto al biofermento preparados con biol y enriquecido con leche y jugo de caña de azúcar, este no cumple con los rangos de necesidades requeridos en los cultivos de caña de azúcar, es decir ningún elemento presente cumple con este. 
Es posible obtener bioles enriquecidos en procesos de fermentación semi-continuos, para ser utilizados en el cultivo de caña de azúcar, pero se hace necesarios seguir realizando estudios con diferentes recetas modificadas.

\section{RECOMENDACIONES}

Se necesitan realizar estudios y pruebas en los biodigestores semi-continuos, con otras recetas modificadas, para obtener biol que cumpla el mayor número de elementos que estén dentro del rango de requerimiento para el cultivo de caña de azúcar.

De igual forma, realizar estudios y pruebas en otro tipo de biodigestores, como son: los modulares, tipo laguna, tipo hindú, etc.; que permita enriquecer los datos existentes en beneficio de los productores.

Es recomendable complementar la investigación, con estudios de los biosoles que se generan en cada uno de los diferentes tipos de biodigestores y los procesos con los que se esté trabajando, ampliando la comparación nutricional de otros cultivos.

\section{BIBLIOGRAFÍA}

Aparcana Robles, S., \& Jansen, A. (2008). Estudio sobre el valor fertilizante de los productos del proceso "Fermentación Anaeróbica" para producción de biogás. Lima, Perú: GermanProfEC-Perú SAC.

Carhuancho León, F. (2012). Aprovechamiento del estiercol de gallina para la elaboración de biol en biodigestores tipo Batch como propuesta al manejo de residuo avicola. Lima, Perú: Universidad Nacional Agraria "La Molina".

CORPOICA. (2008). Uso de microorganismos con potencial de biofertilizante en el cultivo de Mora. Bobota, Col.: Produmedios.

García Jonguitud, K. L. (2020). Evaluación del potencial revalorizable del estiércol de ganado bovino, en un rancho ganadero en Ciudad Valles, S.L.P. Ciudad Valles,S.L.P., Méx.: TecNM campus Ciudad Valles.

Guzmán Flores, J. (2018). Fertilizantes químicos y biofertilizantes en México. Ciuda de México: Camara de Diputados, LXIII Legislatura.

Hernández Medina, A. L. (2021). Tratamiento de estiércol de ganado bovino a través de fermentación homoláctica para obtención de biofertilizante. Ciudad Valles, S.L.P., Méx.: TecNM campus Ciudad Valles.

Kandler, O., \& Van Leeuwenhoek, A. (1983). Carbohydrate metabolism in lactic acid bacteria. Springer Nature, 209-224.

Kinck, A. (1988). Fertilizantes y Fertilización. Barcelona, España: Reverte.

Leveau, J.-Y., \& Bouix, M. (2000). Microbiología Industrial: Los Microorganismos de Interés Industrial. Zaragoza, España: Acribia, Editorial, S.A.

Lindgren, S., \& Dobrogosz, W. (1990). Antagonistic activities of lactic acid bacteria in food and feed fermentations. FEMS Microbiology Review, 149-164. 
McCray, J., \& Mylavarapu, R. (2021). SUGARCANE NUTRIENT MANAGEMENT USING LEAF ANALYSIS. Askifas Powered by Edis, 1-4.

Pacheco Rodríguez, F., Borrero González, G. P., \& Villalobos Rodríguez, M. (2017). Evaluación de la calidad bioquímica resultante de biofermentos agrícolas para uso de familias productoras orgánicas. San José, Costa Rica: Red de Coordinación en Biodiversidad.

Peralta Veran, L., Juscamaita Morales, J., \& Meza Contreras, V. (2016). Obtención y caracterización de abono orgánico líquido a través del tratamiento de excretas del ganado vacuno de un establo lechero usando un consorcio microbiano ácido láctico. Ecologia Aplicada, 1-10.

Restrepo Rivera, J., \& Hensel, J. (2013). El ABC de la agricultura organica, fosfitos y panes de piedra. Calí, Colombia: Feriva.

SENER. (2008). Energía Biomasa - Energías Renovables. Buenos Aires, Argentina: Secretaria de Energía.

Vidal Becerra, E., Mojica Mesinas, C., Acosta Pintor, D. C., \& García Jonguitud, K. L. (2020). Estudio comparativo de biol I (primera generación) y biol II (segunda generación). Ciudad Valles, S.L.P., Méx.: TecNM campus Ciudad Valles. 